\title{
Endometrial histopathology results and evaluation of endometrial cancer risk in geriatric women
}

\author{
Emre Günakan, Zeliha Atak, Mustafa Albayrak, Yüksel Kurban, Gülçin G. Şimşek \\ Department of Obstetrics and Gynecology, Keçiören Training and Research Hospital, University of Medical Sciences, Ankara, Turkey
}

\begin{abstract}
The incidence of premalignant and malignant endometrial disorders increases during the postmenopausal period. In the literature, endometrial disorders are usually discussed in the context of menopausal status. But there are limited data regarding endometrial disorders in geriatric patients. Early diagnosis of endometrial cancers with aggressive behaviour that increases during the geriatric period may allow simpler treatment options and also decrease the treatment-associated morbidity risk.

Records of geriatric patients who underwent an endometrial histopathological evaluation between 2011 and 2016 were evaluated. Clinical findings, transvaginal ultrasonography findings, endometrial sampling methods, and histopathological results were evaluated.

A total of 188 patients were included in the study (mean age $70.3 \pm 5.6$ years). The most common histopathological results were endometrial polyp, atrophic endometrium, and surface epithelium $(26.6 \%, 22.3 \%$, and $12.8 \%$, respectively). None of the 57 patients without vaginal bleeding had endometrial cancer. In 131 patients with vaginal bleeding, mean endometrial thickness was $9.8 \pm 8.1 \mathrm{~mm}(2-49 \mathrm{~mm})$ and the rate of endometrial disorders was $56.5 \%$ (74 patients). Endometrial cancer was diagnosed in 19 patients (10.1\%), and 36.8\% of them had non-endometrioid cancers. The presence of vaginal bleeding was significantly associated with the diagnosis of endometrial cancer and any endometrial disorder ( $p=0.001$ and $p=0.000$, respectively).

The incidence of non-endometrioid endometrial cancers increased in the geriatric period. An endometrial histopathological examination should be considered, especially for patients with a history of vaginal bleeding. Further investigation of the endometrial thickness cut-off levels in the geriatric period will contribute to the literature.
\end{abstract}

Key words: endometrial cancer, endometrial histopathology, geriatric age.

\section{Introduction}

The incidence of premalignant and malignant endometrial disorders increases in the postmenopausal period. Although vaginal bleeding is the most common symptom, patients may present with different symptoms, such as leucorrhoea, post-coital bleeding, or spotting and pelvic pain, or have an asymptomatic course.

Transvaginal ultrasonography (TvUSG) is an acceptable non-invasive method with clinical applicability for endometrial evaluation, and it shows good correlation with pathology results [1]. However, the cut-off value with respect to endometrial thickness in literature is still controversial. Endometrial biopsy is the gold standard method for distinguishing normal endometrium from pathological endometrium. Endometrial biopsy, dilation and curettage (D\&C), and hysteroscopy are the most common endometrial sampling methods.

The most important disorder to be excluded is endometrial cancer. The endometrioid type is the most frequent histological type and has a good prognosis. Non-endometrioid types of endometrial cancer have an increasing trend after the sixth and seventh decades of life and have a poor prognosis.

In our study, we evaluated the endometrial pathological results and clinical findings of geriatric women.

\section{Material and methods}

The records of geriatric patients (over 65 years of age) who underwent endometrial histopathological examination between 2011 and 2016 were retrospectively evaluated. Symptoms, ultrasonographic and clinical examination findings, demographic characteristics, and pathology reports were acquired from patient records. Indications of endometrial biopsy were postmenopausal vaginal bleeding, abnormal endometrial thickness, or fluid seen on TVUSG. Sonographic measurement of endometrial thickness was performed on a sagittal sec- 
tion in an anteroposterior dimension between the basal layers of endometrium. The fluid in the cavity was excluded from the measurement.

Endometrial tissue was obtained during hysterectomy (using abdominal or vaginal approach) or by endometrial sampling (by endometrial biopsy, D\&C, or hysteroscopy). Histopathological results were grouped according to the presence or absence of endometrial cancer or any endometrial disorder (such as polyp, hyperplasia, or endometritis). Endometrial thickness cutoff values between 3 and $5 \mathrm{~mm}$ were used for comparison.

The data were analysed using SPSS version 15.0 (SPSS Inc., Chicago, IL, USA). Descriptive and frequency analyses were performed. Cross-tab analyses were used for sensitivity and specificity calculations. Categorical variables were compared using the $\chi^{2}$ test or Fisher's exact test, where appropriate. A receiver operating characteristic (ROC) curve was used to assess the discriminative role of the endometrial thickness in diagnosis of cancer or any endometrial disorder. The point at which the sum of specificity and sensitivity reached the maximum was taken as the best cut-off point. The cut-off point for endometrial thickness was calculated for all patients, and patients with vaginal bleeding, separately. A $p$-value $<0.05$ was considered statistically significant.

\section{Results}

A total of 188 patients were included in the study. The general characteristics of patients are shown in Table 1 . The mean age of the study population was 70.3 \pm 5.6 years. The most common complaint was vaginal bleeding (131 patients, 69.7\%), and endometrial D\&C was the most common endometrial sampling method (133 patients, $70.7 \%$ ).

Table 2 summarises the histopathological results. The most common histopathological results were endometrial polyp, atrophic endometrium, and surface epithelium (26.6\%, $22.3 \%$, and $12.8 \%$, respectively). There were 57 patients without vaginal bleeding with a mean endometrial thickness of $6 \pm 3 \mathrm{~mm}$ (1-14 mm); 13 of these patients had an endometrial disorder and none had cancer. Vaginal bleeding was present in 131 patients with a mean endometrial thickness of $9.8 \pm 8.1$ $\mathrm{mm}$ (2-49 mm); 74 of these patients (56.5\%) had an endometrial disorder.

Area under the ROC curve was found statistically significant for the discriminative role of endometrial thickness for diagnosis of any endometrial disorder in patients with vaginal bleeding (AUC $=0.789,95 \% \mathrm{Cl}$ : 0.710-0.867, $p<0.001$ ). The best cut-off point for endometrial thickness for predicting the presence of any endometrial disorder was determined as $9.5 \mathrm{~mm}$. The sensitivity rate, specificity rate, and positive and nega- tive predictive values were found to be $57.1 \%, 87.7 \%$, $82.7 \%$, and $60.8 \%$, respectively. The diagnostic accuracy rate was $82.5 \%$ at this point.

For the endometrial thickness cut-off limits of 3, 4, and $5 \mathrm{~mm}$, the sensitivity for the diagnosis of endometrial cancer was $94.7 \%, 78.9 \%$, and $68.4 \%$, respectively. However, the sensitivity for any endometrial disorder was $97.7 \%, 93.1 \%$, and $88.5 \%$, respectively. In subgroup analyses, in patients with vaginal bleeding, sensitivity did not differ for endometrial cancer, whereas it increased for the detection of endometrial disorders, being $98.6 \%, 94.6 \%$, and $89.2 \%$, respectively (Table 3 ).

Table 4 summarises the distribution of cancer cases. Endometrial cancer was diagnosed in 19 patients (10.1\%). Non-endometrioid cancers were diagnosed in seven patients (36.8\% of cancers), and the most frequent type was papillary serous carcinoma (5 patients). Other cancers were squamous cell carcinoma and undifferentiated carcinoma with a carcinosarcomatous component. Adjuvant chemotherapy and/or radiotherapy was required for all of the non-endometrioid cancer patients. On the other hand, endometrial cancer

Table 1. General properties of patients

\begin{tabular}{lcc}
\hline Parameter & Mean & Range or $n$ (\%) \\
\hline Age (years) & $70.53 \pm 5.6$ & $65-90$ \\
\hline Endometrial thickness $(\mathrm{mm})$ & $8.75 \pm 7.2$ & $1-49$ \\
\hline Complaint & \\
\hline Asymptomatic & $42(22.3)$ \\
\hline Vaginal bleeding & $128(69.7)$ \\
\hline Pelvic pain & $9(4.8)$ \\
\hline Leucorrhoea & $2(1.1)$ \\
\hline Ovarian cyst & $4(2.1)$ \\
\hline Endometrial sampling method & $133(70.7)$ \\
\hline Dilation and curettage & $31(16.5)$ \\
\hline Hysteroscopy & $24(12.8)$ \\
\hline Hysterectomy & \\
\hline
\end{tabular}

Table 2. Histopathological results

\begin{tabular}{lccc}
\hline & $\begin{array}{c}\text { With } \\
\text { vaginal } \\
\text { bleeding, } n\end{array}$ & $\begin{array}{c}\text { Without } \\
\text { vaginal } \\
\text { bleeding, } n\end{array}$ & $\begin{array}{c}\text { Total, } \\
n(\%)\end{array}$ \\
\hline Endometrial atrophy & 20 & 22 & $42(22.3)$ \\
\hline Endometrial hyperplasia & 8 & 0 & $8(4.3)$ \\
\hline Endometrial cancer & 19 & 0 & $19(10.1)$ \\
\hline Endometrial polyp & 39 & 11 & $50(26.6)$ \\
\hline Surface epithelium & 16 & 8 & $24(12.8)$ \\
\hline Proliferative & 12 & 9 & $21(11.2)$ \\
endometrium & 9 & 4 & $13(6.9)$ \\
\hline Mucoid material & 6 & 2 & $8(4.3)$ \\
\hline Cervical polyp & 2 & 0 & $2(1.1)$ \\
\hline Endometritis & 0 & 1 & $1(0.5)$ \\
\hline Insufficient sample & & &
\end{tabular}


Table 3. Sensitivity and specificity rates compared with endometrial thickness cut-off levels

\begin{tabular}{|c|c|c|c|c|c|c|}
\hline & \multicolumn{6}{|c|}{ Endometrial thickness } \\
\hline & \multicolumn{2}{|c|}{$3 \mathrm{~mm}$} & \multicolumn{2}{|c|}{$4 \mathrm{~mm}$} & \multicolumn{2}{|c|}{$5 \mathrm{~mm}$} \\
\hline & $\begin{array}{c}\text { Sensitivity } \\
(\%)\end{array}$ & $\begin{array}{c}\text { Specificity } \\
(\%)\end{array}$ & $\begin{array}{c}\text { Sensitivity } \\
(\%)\end{array}$ & $\begin{array}{c}\text { Specificity } \\
(\%)\end{array}$ & $\begin{array}{c}\text { Sensitivity } \\
(\%)\end{array}$ & $\begin{array}{l}\text { Specificity } \\
(\%)\end{array}$ \\
\hline Endometrial cancer & 94.7 & 9.0 & 78.9 & 51.3 & 68.4 & 28.4 \\
\hline Any endometrial disorder & 97.7 & 9.0 & 93.1 & 21.3 & 88.5 & 28.7 \\
\hline $\begin{array}{l}\text { Any endometrial disorder } \\
\text { (with vaginal bleeding) }\end{array}$ & 98.6 & 12.3 & 34.6 & 31.6 & 88.5 & 47.4 \\
\hline
\end{tabular}

Table 4. Distribution of endometrial cancers

\begin{tabular}{lcc}
\hline & $n(\%)$ & Total $n$ (\%) \\
\hline Endometrioid type & & \\
\hline Grade 1 & $7(36.8)$ & $12(63.2)$ \\
\hline Grade 2 & $5(26.3)$ & \\
\hline Non-endometrioid type & & \\
\hline Papillary serous carcinoma & $5(26.3)$ & $7(36.8)$ \\
\hline Squamous cell carcinoma & $1(5.3)$ & \\
\hline Undifferentiated & $1(5.3)$ & \\
\hline
\end{tabular}

was diagnosed in nine of 1219 patients who were under 65 years old. The final histopathology result of one patient could not be reached, but all of the other eight patients (88.8\%) had endometrioid type endometrial cancer in final histopathology.

Moreover, ROC curve analysis was performed to evaluate the discriminative role of endometrial thickness for diagnosis of endometrial cancer in all patients. A cut-off of $11.5 \mathrm{~mm}$ was found (AUC $=0.616,95 \% \mathrm{Cl}$ : $0.445-0.788, p=0.097)$. In $\chi^{2}$ test analyses the sensitivity rate, specificity rate, positive and negative predictive values, and accuracy rate were found to be $57.9 \%$, $83.4 \%, 28.2 \%, 94.6 \%$, and $80.9 \%$, respectively, with $p$-value $<0.001$.

All 19 patients with endometrial cancer had a history of vaginal bleeding. The presence of vaginal bleeding was significantly associated with the diagnosis of endometrial cancer and any endometrial disorder ( $p=0.001$ and $p=0.000$, respectively).

\section{Discussion}

Endometrial sampling is performed to exclude endometrial cancer in women during the postmenopausal period. Surgical treatment is often required for premalignant or malignant endometrial diseases. Complicated medical or surgical treatment options can be avoided by detecting these disorders at an early stage.

TVUSG is the most frequently used non-invasive endometrial evaluation method. With a high negative predictive value for the differentiation of uterine and ovarian disorders in postmenopausal patients [1], and it can be safely and effectively used as the first step of evaluation [2]. Saline infusion sonography (SIS) may be more useful than TVUSG for small focal lesions (such as polyps and submucosal fibroids), which can be missed on TVUSG [3]. Computed tomography and magnetic resonance imaging can also be used for imaging of the female genital tract for a differential diagnosis.

In published data, because there is no clear consensus regarding what constitutes abnormal endometrial thickness, further investigation is required. Not only endometrial thickness but also the initial complaint of the patient, clinical examination findings, and experience of the clinician are relevant for management. In a woman with postmenopausal bleeding, the Society of Radiologists in Ultrasound advises that TVUSG (with an endometrial thickness of $\leq 5 \mathrm{~mm}$ ) or endometrial sampling is effective as a first diagnostic step [2]. A metaanalysis including 13 studies of 2896 women reported that the sensitivity of TVUSG for detecting endometrial cancer for endometrial thickness of 4 and $5 \mathrm{~mm}$ was $95 \%$ and $90 \%$, respectively; at $3 \mathrm{~mm}$, the sensitivity was 98\% [4]. The American College of Obstetricians and Gynaecologists reported that if the endometrial thickness is $<4 \mathrm{~mm}$ on TVUSG in patients with postmenopausal bleeding, endometrial sampling is not required [5]. On the other hand, for postmenopausal women without bleeding, Smith-Bindman et al. suggested that if the endometrial thickness is > $11 \mathrm{~mm}$, the cancer risk is $6.7 \%$ and a biopsy should be considered, whereas if the endometrial thickness is $\leq 11 \mathrm{~mm}$, the risk of cancer is very low [6]. The reason for endometrial fluid is usually stenosis of the cervix in the postmenopausal period, but concomitant endometrial thickening $>3 \mathrm{~mm}$ is related to an increased risk of endometrial cancer. A normal endometrium of $<3 \mathrm{~mm}$ on ultrasound in patients without vaginal bleeding does not require further investigation [7].

For evaluating postmenopausal bleeding, an endometrial biopsy is the preferred initial test owing to its high sensitivity, low complication rate, low cost, and easy clinical applicability. However, in patients at high risk of endometrial cancer with a non-diagnostic or insufficient pathology specimen, D\&C may provide a better evaluation. Furthermore, hysteroscopic biopsy is a useful technique for focal lesions that can be missed by $D \& C[8]$. 
Endometrial cancer is the most common gynaecological cancer in developed countries [9]. The incidence increases after the fifth decade of life. Type 1 endometrial cancer includes the low-grade endometrioid histological type that is usually caught at an early stage and has a very good prognosis.

Type 2 endometrial cancer includes non-endometrioid, such as serous or clear cell carcinoma, and highgrade endometrioid types and shows an increasing incidence after the sixth and seventh decades of life. These usually develop in the ground of atrophic endometrium and are diagnosed at an advanced stage. Initial treatment includes surgery followed by adjuvant chemotherapy. This group constitutes $10-20 \%$ of endometrial cancers and $40 \%$ of deaths related to endometrial cancers $[10,11]$. Owing to the poor patient performance in geriatric age, the risk of morbidity related to surgery or adjuvant treatment increases, but this can be avoided with an early diagnosis after performing an appropriate endometrial evaluation.

In our study, we determined that there is a high incidence of endometrial cancer $(10.1 \%)$ in geriatric women. Cancer was detected only in patients with vaginal bleeding. We also found a higher incidence of non-endometrioid endometrial cancers (36.8\%) in this age group compared with under geriatric age. A cut-off point of $9.5 \mathrm{~mm}$ for endometrial thickness was found to be significantly associated with the detection of any endometrial disorder in patients with vaginal bleeding. A cut-off point of $11.5 \mathrm{~mm}$ for endometrial thickness had a low AUC value despite its high negative predictive value (94.6\%). The sensitivity of a cut-off value of $3 \mathrm{~mm}$ for endometrial thickness was $94.7 \%$ for endometrial cancer, similar to other reports in the literature, but owing to our limited patient population, we do not suggest any cut-off level for endometrial thickness.

\section{Conclusions}

Published data on postmenopausal endometrial disorders mostly include the whole postmenopausal period. However, the patient profiles and endometrial disorder types differ in the geriatric age group. The incidence of non-endometrioid endometrial cancers is increased in the geriatric period. We suggest that endometrial histopathological evaluation should be considered in geriatric patients with a history of vaginal bleeding. Further studies regarding the endometrial thickness cut-off levels in the geriatric period will contribute to the literature.

\section{Disclosure}

Ethical approval for the present study was not required because it was retrospectively performed.

Authors report no conflict of interest.

\section{References}

1. Lee JH, Dubinsky T, Andreotti RF, et al. ACR Appropriateness Criteria ${ }^{\circledR}$ assessment of gravid cervix. Ultrasound Q 2011; 27: 275-280.

2. Goldstein RB, Bree RL, Benson CB, et al. Evaluation of the woman with postmenopausal bleeding: Society of Radiologists in Ultrasound-Sponsored Consensus Conference statement. J Ultrasound Med 2001; 20: 1025-1036.

3. Kamel HS, Darwish AM, Mohamed SA. Comparison of transvaginal ultrasonography and vaginal sonohysterography in the detection of endometrial polyps. Acta Obstet Gynecol Scand 2000; 79: 60-64.

4. Timmermans A, Opmeer BC, Khan KS, et al. Endometrial thickness measurement for detecting endometrial cancer in women with postmenopausal bleeding: a systematic review and meta-analysis. Obstet Gynecol 2010; 116: 160-167.

5. ACOG Committee Opinion No. 440. American College of Obstetricians and Gynecologists. The role of transvaginal ultrasonography in the evaluation of postmenopausal bleeding. Obstet Gynecol 114: 409-411.

6. Smith-Bindman R, Weiss E, Feldstein V. How thick is too thick? When endometrial thickness should prompt biopsy in postmenopausal women without vaginal bleeding. Ultrasound Obstet Gynecol 2004; 24: 558-565.

7. Debby A, Malinger G, Glezerman M, Golan A. Intra-uterine fluid collection in postmenopuasal women with cervical stenosis. Maturitas 2006; 55: 334-337.

8. Epstein E, Ramirez A, Skoog L, et al. Dilatation and curettage fails to detect most focal lesions in the uterine cavity in women with postmenopausal bleeding. Acta Obstet Gynecol Scand 2001; 80: 1131-1136.

9. Ferlay J, Soerjomataram I, Dikshit R, et al. Cancer incidence and mortality worldwide: sources, methods and major patterns in GLOBOCAN 2012. Int J Cancer 2015; 136: E359-E386.

10. Kurman RJ, Scully RE. Clear cell carcinoma of the endometrium: an analysis of 21 cases. Cancer 1976; 37: 872-882.

11. Zorn KK, Bonome T, Gangi L, et al. Gene expression profiles of serous, endometrioid, and clear cell subtypes of ovarian and endometrial cancer. Clin Cancer Res 2005; 11: 6422-6430. 